\title{
Switching Magnetism and Superconductivity with Spin-Polarized Current in Iron-Based Superconductor
}

\author{
Seokhwan Choi, ${ }^{1}$ Hyoung Joon Choi, ${ }^{2}$ Jong Mok Ok, ${ }^{3,4}$ Yeonghoon Lee, ${ }^{1}$ Won-Jun Jang, ${ }^{1,5, \uparrow}$ \\ Alex Taekyung Lee, ${ }^{6}$ Young Kuk, ${ }^{7}$ SungBin Lee, ${ }^{1}$ Andreas J. Heinrich, ${ }^{8,9}$ Sang-Wook Cheong, ${ }^{10}$ \\ Yunkyu Bang, ${ }^{11}$ Steven Johnston, ${ }^{12}$ Jun Sung Kim, ${ }^{3,4}$ and Jhinhwan Lee ${ }^{1, *}$ \\ ${ }^{1}$ Department of Physics, Korea Advanced Institute of Science and Technology, Daejeon 34141, Korea \\ ${ }^{2}$ Department of Physics and Center for Computational Studies of Advanced Electronic Material Properties, \\ Yonsei University, Seoul 03722, Korea \\ ${ }^{3}$ Department of Physics, Pohang University of Science and Technology, Pohang 37673, Korea \\ ${ }^{4}$ Center for Artificial Low Dimensional Electronic Systems, Institute for Basic Science, Pohang 37673, Korea \\ ${ }^{5}$ Center for Axion and Precision Physics Research, Institute for Basic Science (IBS), Daejeon 34051, Korea \\ ${ }^{6}$ Department of Applied Physics and Applied Mathematics, Columbia University, New York, New York 10027, USA \\ ${ }^{7}$ Department of Physics and Astronomy, Seoul National University, Seoul 08826, Korea \\ ${ }^{8}$ Center for Quantum Nanoscience, Institute for Basic Science (IBS), Seoul 03760, Korea \\ ${ }^{9}$ Physics Department, Ewha Womans University, Seoul 03760, Korea \\ ${ }^{10}$ Rutgers Center for Emergent Materials and Department of Physics and Astronomy, \\ Rutgers University, Piscataway, New Jersey 08854, USA \\ ${ }^{11}$ Department of Physics, Chonnam National University, Gwangju 61186, Korea \\ ${ }^{12}$ Department of Physics and Astronomy, University of Tennessee, Knoxville, Tennessee 37996-1200, USA \\ (Received 19 June 2017; revised manuscript received 8 September 2017; published 27 November 2017)
}

We explore a new mechanism for switching magnetism and superconductivity in a magnetically frustrated iron-based superconductor using spin-polarized scanning tunneling microscopy (SPSTM). Our SPSTM study on single-crystal $\mathrm{Sr}_{2} \mathrm{VO}_{3} \mathrm{FeAs}$ shows that a spin-polarized tunneling current can switch the Fe-layer magnetism into a nontrivial $C_{4}(2 \times 2)$ order, which cannot be achieved by thermal excitation with an unpolarized current. Our tunneling spectroscopy study shows that the induced $C_{4}(2 \times 2)$ order has characteristics of plaquette antiferromagnetic order in the Fe layer and strongly suppresses superconductivity. Also, thermal agitation beyond the bulk Fe spin ordering temperature erases the $C_{4}$ state. These results suggest a new possibility of switching local superconductivity by changing the symmetry of magnetic order with spin-polarized and unpolarized tunneling currents in iron-based superconductors.

DOI: 10.1103/PhysRevLett.119.227001

Iron-based superconductors (FeSCs) have shown intriguing phenomena related to the coexistence of magnetism and superconductivity below the superconducting transition temperature $\left(T_{c}\right)$ [1-3]. Although an understanding of their detailed interplay is still under debate, certain magnetic orders seem to be very crucial in realizing coexistent superconductivity [3-15]. Recent studies have shown new reentrant $C_{4}$ symmetric antiferromagnetic phases $\left(C_{4}\right.$ magnetism from now on) coexisting with superconductivity and have reported that the superconducting $T_{c}$ is suppressed by $C_{4}$ magnetic order [16-19]. Direct atomic-scale control of the Fe layer's magnetic symmetry and the determination of its correlation with superconductivity may be useful for an in-depth understanding of the interplay between superconductivity and magnetism. To our knowledge, there has been no report of a direct realspace observation of such a control by local probes and atomic-scale demonstration of the correlation of magnetism and superconductivity.

In this regard, the parent compound tetragonal ironbased superconductor $\mathrm{Sr}_{2} \mathrm{VO}_{3} \mathrm{FeAs}$ with $T_{c} \approx 33 \mathrm{~K}[20]$ is an ideal candidate where the interplay between magnetism and superconductivity can be directly demonstrated due to its nearly degenerate magnetic ground states. $\mathrm{Sr}_{2} \mathrm{VO}_{3} \mathrm{FeAs}$ has two types of square magnetic ion lattices: a square $\mathrm{Fe}$ lattice in the FeAs layer and a square $\mathrm{V}$ lattice in the two neighboring $\mathrm{VO}_{2}$ layers. At optimal doping, the FeAs layer usually prefers $C_{2}$ magnetism harboring superconductivity, while the $\mathrm{VO}_{2}$ layer prefers $C_{4}$ magnetism [1-3,21]. Previous experimental studies of $\mathrm{Sr}_{2} \mathrm{VO}_{3} \mathrm{FeAs}$ [22-28], however, have reported inconsistent results about magnetic order; recent nuclear magnetic resonance (NMR) measurements on single crystals [29] and neutron diffraction [30] experiments show that there is no long-range magnetic order in the $\mathrm{V}$ lattice at any temperature, while in the Fe lattice a magnetic order with a small moment of $\sim 0.05 \mu_{B}$, possibly due to frustration, is developed below $50 \mathrm{~K}$. Indeed, a theoretical generalized gradient approximation (GGA) calculation has suggested that there can be a number of competing metastable magnetic states composed of different symmetries in V and Fe layers [21]. This is a reasonable theoretical prediction considering the coupling 
and frustration between $\mathrm{V}$ and $\mathrm{Fe}$ layers (Supplemental Material Sec. II [31]). Therefore, it has been quite a challenging and interesting experimental task to determine the possible magnetic ground states of the heterostructure superconductor $\mathrm{Sr}_{2} \mathrm{VO}_{3} \mathrm{FeAs}$ and the possible methods to adjust their balances.

One possible way to explore the potentially frustrated magnetic states and their relation to superconductivity is using a spin-polarized scanning tunneling microscope (SPSTM) to locally modify the magnetic environment with a spin-polarized tunneling current. Our density functional theory (GGA) calculation (Supplemental Material Sec. I [31]) showed a possibility that a nonzero net spin density by the injection of a spin-polarized tunneling current can induce a $C_{4}$ magnetic order from a pristine $C_{2}$ magnetic order due to the Hund interaction, as illustrated schematically in Figs. 1(a)-1(d). The spin transfer torque and Joule heating effects will then provide the energies to overcome the characteristic potential barriers between the different magnetic states [33].

In this Letter, using a SPSTM we demonstrate that a spin-polarized tunneling current can induce a nontrivial metastable $C_{4}$ magnetic order in the Fe layer not usually achievable through thermal excitation. We also show that a thermal annealing beyond the bulk Fe magnetic ordering temperature erases the induced $C_{4}$ magnetic order. From the tunneling spectroscopy analysis measured inside and outside of the region of the induced $C_{4}$ magnetic order, we
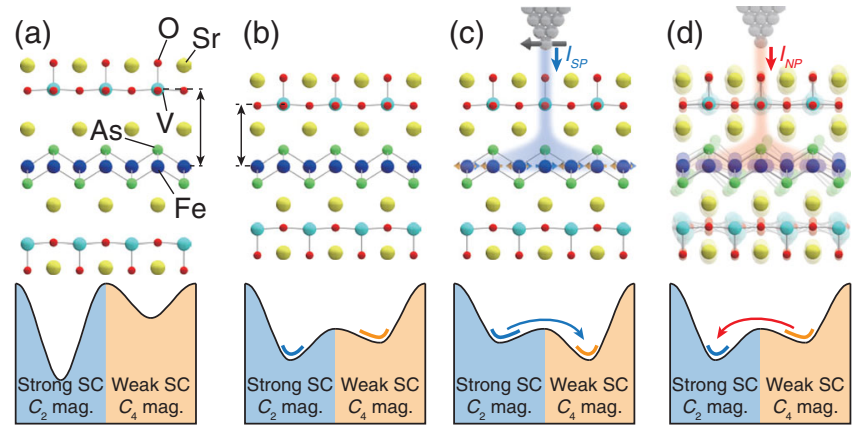

FIG. 1. (a)-(d) Schematic illustrations of FeAs-layer configuration potential landscapes for $\mathrm{Sr}_{2} \mathrm{VO}_{3} \mathrm{FeAs}$ in various situations. (a) The imaginary case of $\mathrm{FeAs}$ and $\mathrm{Sr}_{2} \mathrm{VO}_{3}$ layers being separated sufficiently apart while the electron doping from the $\mathrm{Sr}$ layer retained near optimal. The $C_{2}$ magnetism in the Fe layer with strong superconductivity is preferred. (b) The natural separation found in $\mathrm{Sr}_{2} \mathrm{VO}_{3} \mathrm{FeAs}$ results in interlayer coupling and near degeneracy among the magnetic states with different symmetries, with the $C_{2}$ magnetism with strong superconductivity still being the ground state. (c) If a sufficiently strong spin-polarized current is injected, the balances among these states may change, possibly resulting in $C_{4}$ magnetic states with weak superconductivity in the FeAs layer. (d) When the sample is thermally annealed globally or locally with a high bias tunneling current injection, it may return to the ground states with $C_{2}$ magnetism and strong superconductivity. also find a signature of suppressed superconductivity in the $C_{4}$ order region, which is shown to be consistent with the nesting and spin fluctuation scenario of iron-based superconductivity.

We grew single crystals of $\mathrm{Sr}_{2} \mathrm{VO}_{3} \mathrm{FeAs}$ with a self-flux method [29], which are then cleaved in situ at a temperature $\sim 15 \mathrm{~K}$ just before mounting on the STM head. Because of the weakly van der Waals-coupled SrO-SrO layers, the cleaved surface is almost always terminated with a symmetrically cleaved $\mathrm{SrO}$ layer. For real-space magnetic imaging and injection of a spin-polarized current, we have developed a technique of SPSTM with an antiferromagnetic Cr-cluster tip (Cr tip, from now on), which is created in situ on a $\mathrm{Cr}(001)$ surface (Supplemental Material Sec. III [31]). Each $\mathrm{Cr}$ tip is confirmed on $\mathrm{Cr}(001)$ steps for spin contrasts [Fig. S2(c)] and no gap in the $d I / d V$ spectrum on $\mathrm{Cr}(001)$ [Fig. S2(e)].

The 4.6 K STM topographic image of the as-cleaved SrO top layer of $\mathrm{Sr}_{2} \mathrm{VO}_{3} \mathrm{FeAs}$ taken with an unpolarized $\mathrm{W}$ tip in Fig. 2(c) shows small randomly oriented domains of quasi$\mathrm{C}_{2}$-symmetric atomic corrugations. These show no preference for any particular fourfold lattice direction over large scales, consistent with their identity as surface reconstructions (SRs) in the absence of bulk orthorhombicity [30].

In contrast, our SPSTM images with a spin-polarized Cr tip show (above a small bias threshold $[\sim 30 \mathrm{meV}$, $\sim 25 \mathrm{pA}])$, a $C_{4}$ symmetric $(2 \times 2)$ order with intra-unit-cell topographic modulations [Figs. 2(d) and 2(e)] without any signature of SR seen in unpolarized tip images [Fig. 2(c)]. This observation implies that the spin-polarized current induces randomly fluctuating SRs with a flat time average (see Fig. S7). At the same time, any magnetic signal of a Fe layer observed on the top layer oxygen should be the average of the four neighboring Fe spins connected to the As ions in each vertical O-V-As tunneling path as shown in Figs. 2(a) and 2(b). Hence, the most natural explanation for the observed $(2 \times 2)$ pattern with three groups of apparent height levels is the plaquette order in the Fe lattice with flat time-averaged SRs. The Fourier-transformed $q$-space image [the inset in Fig. 2(d)] also shows the double wave vectors $\boldsymbol{Q}=(\pi / 2, \pi / 2)_{\mathrm{Fe}}$ and $\boldsymbol{Q}^{*}=(\pi / 2,-\pi / 2)_{\mathrm{Fe}}$ expected from the plaquette order in Ref. [8].

To understand the nature of magnetic metastability in this system, we performed a comparative study of biasdependent topographic measurements using unpolarized (W) and spin-polarized $(\mathrm{Cr})$ tips at $4.6 \mathrm{~K}$. Using the unpolarized tip, shown in Figs. 3(a)-3(c), we found that the surface starts to change at biases beyond $V_{\text {th }}^{N} \approx$ $300 \mathrm{meV}$ and the fluctuation becomes so rapid above $400 \mathrm{meV}$ that the surface starts to appear essentially flat as a result of time averaging of the fluctuations. Returning to the low bias condition, as shown in Fig. 3(d), we observe that the square area which experienced the high bias scanning has completely changed with sharply defined boundaries, as shown in Fig. 4(a). 


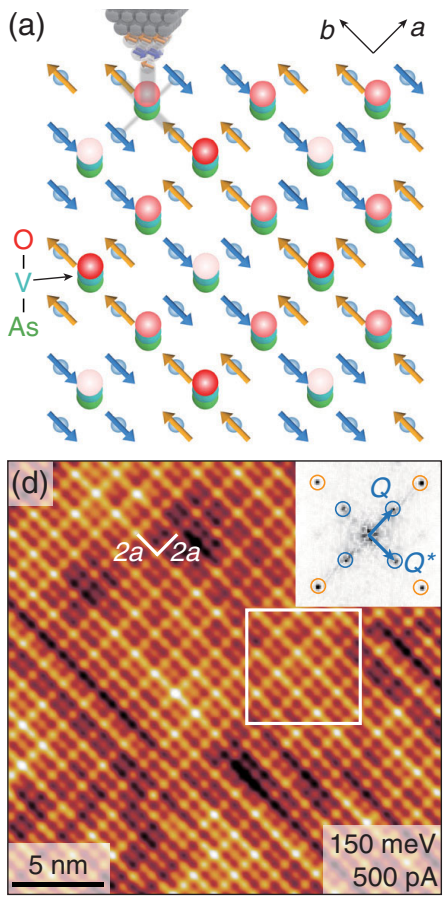

(b)
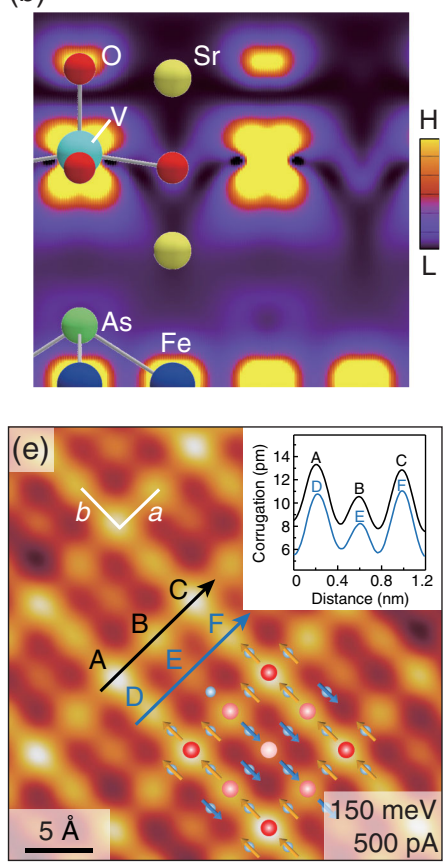
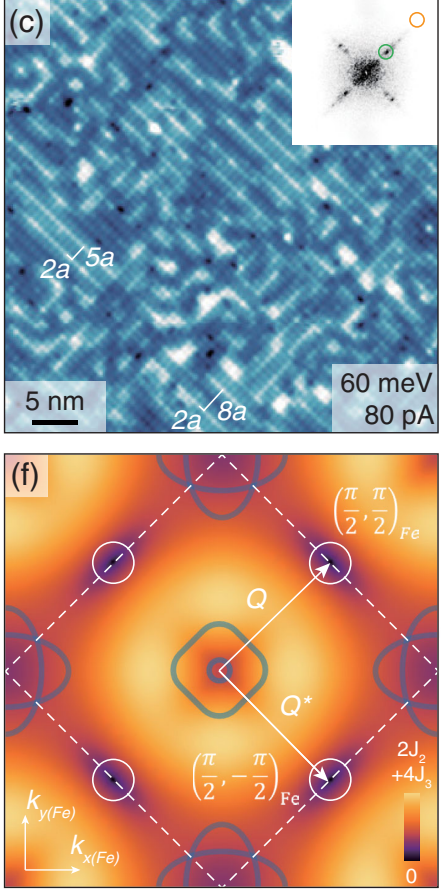

FIG. 2. (a) The structure of the Fe magnetic moments in the $C_{4}$ (plaquette) order. Each red dot represents the oxygen at the top of each vertical O-V-As atomic chain acting as the tunneling path. (b) The theoretical electron density plot near the Fermi level (integrated over $[-50,0] \mathrm{meV})$. (c) A typical $4.6 \mathrm{~K}$ topograph taken with $\mathrm{W}$ tip showing quasi- $C_{2}$ SRs with random orientations. (d) A spin-polarized STM image taken at a low junction resistance with a nearly in-plane polarized $\mathrm{Cr}$ tip showing the induced $C_{4}$-symmetric $(2 \times 2)$ order. The orange, green, and blue circles in the Fourier-transformed images (the insets) in (c) and (d) indicate $|\boldsymbol{q}|=2 \pi / a$ (Bragg peaks), $3 \pi / 4 a$, and $\pi / a$, respectively. (e) The magnified view of the area in a white square in (d), with the $(2 \times 2)$ magnetic unit cells with a $C_{4}$ plaquette spin model overlaid. Its inset shows the cross sections along the black and blue arrows. (f) The spin-wave dispersion of the $C_{4}$ plaquette order and its two momentum transfer vectors $\left(\boldsymbol{Q}\right.$ and $\left.\boldsymbol{Q}^{*}\right)$ from the localized moment picture [8], shown together with the ARPES-based Fermi surfaces (dark curves) [34].

In case of the spin-polarized tip [Figs. 3(e)-3(g)], the SPSTM image is qualitatively identical to the unpolarized tip case at low bias near $10 \mathrm{meV}$, but the surface starts to change beyond a threshold bias $V_{\mathrm{th}}^{\mathrm{SP}} \approx 30 \mathrm{meV}$, revealing the $(2 \times 2)$ domain structure and its phase domain walls. The significantly lower bias threshold voltage for a spinpolarized tip is indicative of the final state and the transition mechanism qualitatively different from those achieved by an unpolarized tip as will be discussed further with Fig. 4. Returning to the very low bias condition shown in Fig. 3(h), we found that the $C_{4}$ order is still retained with extra fluctuations (visible as random horizontal streaks) implying the extra degeneracy in the $C_{4}$ state.

The qualitative equivalence of the pristine surface images taken with an unpolarized tip [Fig. 3(a)] and a spin-polarized tip [Fig. 3(e)] can be understood from the fact that the pristine state will probably have either $C_{2}$ single-stripe correlations or (in the presence of disorder) short-range $C_{2}$ single-stripe orders, both supporting superconducting pairing (Refs. [21,29], and Supplemental Material Sec. I [31]). Neither of these two kinds of $C_{2}$ magnetism is detectable by SPSTM due to the particular tunneling geometry of this material [Fig. S3(d)].
In order to explore the possibility of erasing of the $C_{4}$ order by thermal excitation, we performed a variable temperature Cr-tip SPSTM measurement [Figs. 3(i)-3(1) and Fig. S10]. We found that the $C_{4}$ order can be erased near $60 \mathrm{~K}$, right above the Fe magnetic ordering temperature found in NMR measurement [29]. On the other hand, the application of a magnetic field up to $7 \mathrm{~T}$ does not induce any qualitative change in the $C_{4}(2 \times 2)$ pattern in the Cr tip SPSTM topograph [Fig. S11]. These show that the induced $C_{4}$ order is an antiferromagnetic order in the Fe layer and the switching of the Fe magnetism is reversible by thermal agitation beyond the bulk $\mathrm{Fe}$ magnetic ordering temperature.

To study the connection between superconductivity and the $C_{4}$ magnetic order, we performed a comparative spectroscopic study. We first acquired a large-area topograph using a unpolarized tip with a bias condition below threshold $V_{\mathrm{th}}^{N}$. We then scanned over a smaller square area near the center [black dotted square in Fig. 4(a)] with a bias condition exceeding the threshold $V_{\mathrm{th}}^{N}$, simulating thermal annealing in this area. Figure 4(a) shows the topograph taken immediately afterwards with a bias condition below $V_{\mathrm{th}}^{N}$. It shows the changed surface topographic pattern, 


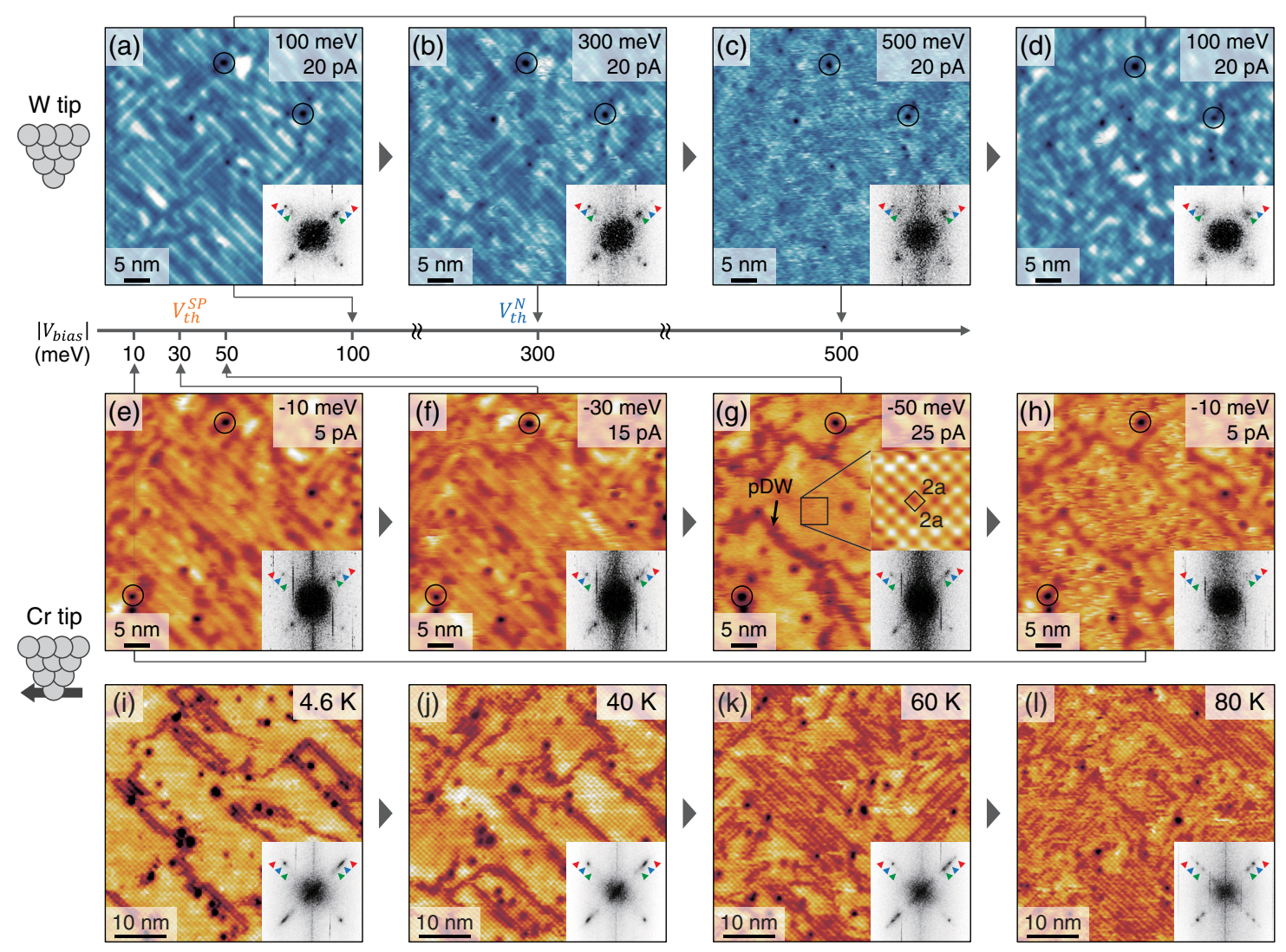

FIG. 3. (a)-(d) Bias dependence of W-tip topograph images showing a threshold voltage for SR fluctuation near $V_{\text {th }}^{N} \approx 300$ meV. (e)-(h) Bias dependence of Cr-tip topograph images. $C_{4}$ symmetric $(2 \times 2)$ domains and phase domain walls $(\mathrm{g})$ are induced at a significantly lower threshold $\left(V_{\mathrm{th}}^{\mathrm{SP}} \approx 30 \mathrm{meV}\right)$, indicating a qualitatively different final state from that obtained with the W tip (d). The inset in (g) is taken at a slightly higher junction conductance $[-50 \mathrm{mV}, 100 \mathrm{pA}]$. In all the FFT insets, the blue (red, green) arrows correspond to $|\boldsymbol{q}|=\pi / a_{0}\left(5 \pi / 4 a_{0}, 3 \pi / 4 a_{0}\right)$. (i)-(l) Temperature-dependent Cr-tip topographs taken at bias [-50 mV, $\left.100 \mathrm{pA}\right]$ showing the erasure of the $C_{4}$ order beyond the Fe magnetic ordering temperature.

which corresponds to another instance of the nearly degenerate ground states achievable by tunneling current-induced nonuniform thermal excitation. Then we measured the $d I / d V$ spectra inside [annealed, Fig. 4(c), blue solid curve] and outside [as-cleaved, Fig. 4(c), green solid curve] of the central high-bias-scanned region. The tunneling spectra measured in both regions identically show various features: a pair of superconducting coherence peaks near -6 and $+6 \mathrm{meV}$ and SDW gap-edge-like features near -18 and $+14 \mathrm{meV}$. These spectral features are virtually independent of the changes in SRs as demonstrated in Supplemental Material of Ref. [31]. This implies that the difference in both regions is only the modification of SR due to thermal agitation by the tunneling current and that most of the spectral features, including the superconducting gap, are the physics in the FeAs layer beneath the topmost $\mathrm{Sr}_{2} \mathrm{VO}_{3}$ layer [35].

In the case of a spin-polarized $(\mathrm{Cr})$ tip, the results are qualitatively different. Figure 4(b) shows a large-area topograph taken with a bias condition below $V_{\text {th }}^{\text {SP }}$ after scanning over the smaller square region (black dotted square) with biases over $V_{\text {th }}^{\mathrm{SP}}$ [Figs. 3(e)-3(g)]. The central square region shows the well-defined $C_{4}$ domains (and various domain walls) induced by the spin-polarized current causing a sustained spin polarization lowering the $C_{4}$ order energy under the tip. The $d I / d V$ spectra measured in the region with $C_{4}$ order [red and purple curves in Fig. 4(d)] show that the superconducting coherence peaks and the SDW-gap-edge-like features are both significantly suppressed in the presence of $C_{4}$ magnetic order.

One plausible explanation for suppressed superconductivity in this particular $C_{4}$ (plaquette) order is related to the mutual relationship of the spin-wave dispersion in Fig. 2(f) (derived from Ref. [8]) and the overlaid Fermi surfaces observed in angle-resolved photoemission spectroscopy (ARPES) measurement [34]. For the $C_{4}$ plaquette order, the low-energy spin fluctuations with wave vectors $\boldsymbol{Q}$ and $Q^{*}$ do not satisfy the nesting condition between any pair of the Fermi surfaces $\Gamma$ and $M(X)$ and thus are unable to effectively mediate pairing in the spin-fluctuation-based theory of iron-based superconductivity. According to this scenario, the suppression of the nesting condition by the induced $C_{4}$ plaquette order will have a more drastic effect on superconductivity compared with switching between $C_{2}$ 

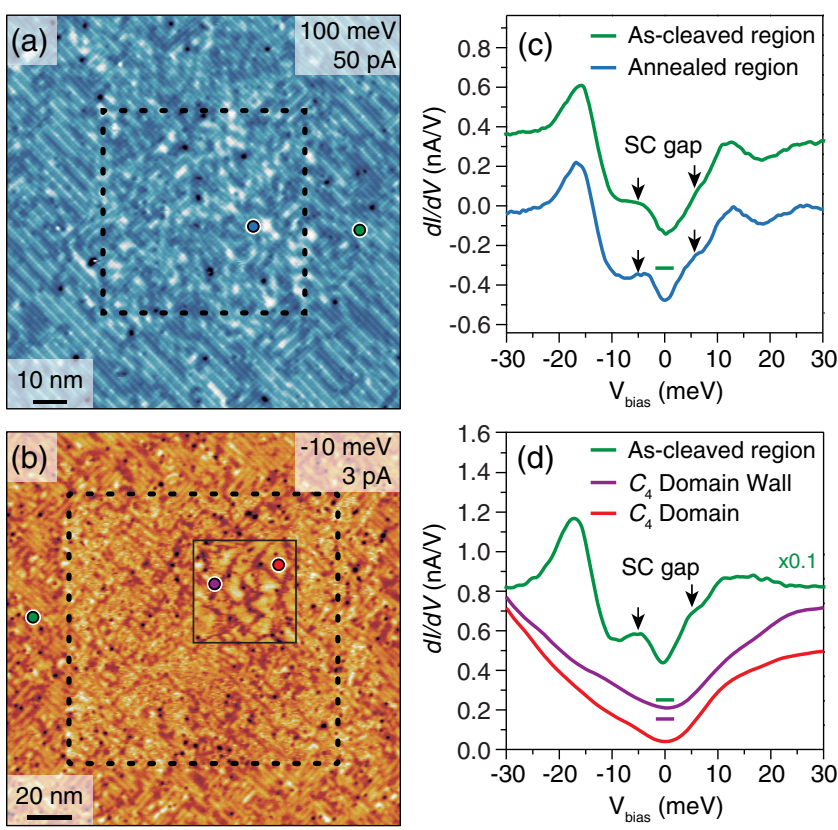

FIG. 4. (a) [(b)] W-tip (Cr-tip) topograph with bias conditions below threshold $V_{\text {th }}^{N}\left(V_{\text {th }}^{\text {SP }}\right)$ taken after the higher bias scans shown in Figs. 3(a)-3(c) [Figs. 3(e)-3(g)] performed only in the area inside the dotted square. The inset image in the solid square in (b) is a Cr-tip topograph with a higher bias above $V_{\text {th }}^{\text {SP }}$ showing the domains and the domain walls more clearly (see Fig. S8). (c) [(d)] shows the tunneling spectra measured at marked positions with corresponding marker colors in (a) with a W tip [(b) with a $\mathrm{Cr}$ tip]. The W-tip spectra were measured at bias [40 meV, $300 \mathrm{pA}$ ]. The Cr-tip spectra were measured at the set point of $[50 \mathrm{meV}$, $120 \mathrm{pA}]$ inside the $C_{4}$ region and at the set point of $[30 \mathrm{meV}$, $5 \mathrm{pA}]$ in the pristine region with a larger averaging time to avoid inducing a $C_{4}$ state during the $d I / d V$ measurement. All the data are taken at $4.6 \mathrm{~K}$.

and $C_{4}$ orders that maintain the nesting conditions, as shown in the recent studies on $\mathrm{Ba}_{1-x} \mathrm{~K}_{x} \mathrm{Fe}_{2} \mathrm{As}_{2}$ [17] and $\mathrm{Ba}_{1-x} \mathrm{Na}_{x} \mathrm{Fe}_{2} \mathrm{As}_{2}$ [18], where a more subtle $T_{c}$ reduction was observed. Among multiple theories of iron-based superconductivity based on spin fluctuations [36,37] and orbital fluctuations [38,39], our experimental results on this material seem to favor the former.

In summary, we carried out a real-space study of correlation between superconductivity and $C_{4}$ magnetism in an iron-based superconductor by changing the magnetic symmetry using spin-polarized STM. In this magnetically frustrated material, a spin-polarized tunneling current induced a nontrivial metastable $C_{4}$ order not usually accessible through thermal excitation, while thermal agitation beyond the bulk $\mathrm{Fe}$ spin ordering temperature erased the $C_{4}$ state. We also observed suppressed superconductivity in the $C_{4}$ order region induced by a spinpolarized current consistent with the spin-fluctuation-based theories. These are a unique and clear demonstration of switching the Fe-layer magnetism and superconductivity by spin-polarized current injection and thermal agitation.
As suggested in Fig. S12, our findings may be extended toward future studies for heterostructure superconductor devices manipulating magnetism and superconductivity using spin-polarized and unpolarized currents.

The authors are thankful for helpful discussions with A. Chubukov, I. Mazin, H.-J. Lee, S.-J. Kahng, Ja-Yong Koo, C. Kim, J. J. Yu, W. Wu, K.-J. Kim, and J. H. Shim. This work was supported by National Research Foundation (NRF) through the Pioneer Research Center Program (No. 2013M3C1A3064455), the Basic Science Research Programs (No. 2017R1D1A1B01016186), SRC Center for Topological Matter (No. 2011-0030785), the Creative Research Initiative Program (No. 2011-0018306), the Max Planck POSTECH/KOREA Research Initiative Program (No. 2016K1A4A4A01922028), and the Brain Korea 21 PLUS Project of Korea Government. It is also supported by IBS-R017-D1 and IBS-R027-D1 and by Korea Research Institute of Standards and Science through the Metrology Research Center Program funded (No. 201515011069), by MSIP of Korea through NRF (2015R1C1A1A01052411), by the Samsung Advanced Institute of Technology (SAIT), and by the Gordon and Betty Moore Foundation's EPiQS Initiative through Grant No. GBMF4413 to the Rutgers Center for Emergent Materials. Computational resources have been provided by KISTI Supercomputing Center (Project No. KSC-2016C3-0052).

*Corresponding author.

jhinhwan@kaist.ac.kr

Present address: Center for Quantum Nanoscience, Institute for Basic Science (IBS), Seoul 03760, Republic of Korea; Department of Physics, Ewha Womans University, Seoul 03760, Republic of Korea.

[1] D. C. Johnston, The puzzle of high temperature superconductivity in layered iron pnictides and chalcogenides, Adv. Phys. 59, 803 (2010).

[2] H. Hosono and K. Kuroki, Iron-based superconductors: Current status of materials and pairing mechanism, Physica C (Amsterdam) 514, 399 (2015).

[3] P. Dai, Antiferromagnetic order and spin dynamics in ironbased superconductors, Rev. Mod. Phys. 87, 855 (2015).

[4] P. Dai, J. Hu, and E. Dagotto, Magnetism and its microscopic origin in iron-based high-temperature superconductors, Nat. Phys. 8, 709 (2012).

[5] P. Chandra, P. Coleman, and A. I. Larkin, Ising Transition in Frustrated Heisenberg Models, Phys. Rev. Lett. 64, 88 (1990).

[6] A. Chubukov, First-order transition in frustrated quantum antiferromagnets, Phys. Rev. B 44, 392 (1991).

[7] J. K. Glasbrenner, I. I. Mazin, H. O. Jeschke, P. J. Hirschfeld, R. M. Fernandes, and R. Valentí, Effect of magnetic frustration on nematicity and superconductivity in iron chalcogenides, Nat. Phys. 11, 953 (2015). 
[8] S. Ducatman, N. B. Perkins, and A. Chubukov, Magnetism in Parent Iron Chalcogenides: Quantum Fluctuations Select Plaquette Order, Phys. Rev. Lett. 109, 157206 (2012).

[9] J. Villain, R. Bidaux, J.-P. Carton, and R. Conte, Order as an effect of disorder, J. Phys. (Paris) 41, 1263 (1980).

[10] F. Ma and Z.-Y. Lu, Iron-based layered compound LaFeAsO is an antiferromagnetic semimetal, Phys. Rev. B 78, 033111 (2008).

[11] J. Dong et al., Competing orders and spin-density-wave instability in $\mathrm{La}\left(\mathrm{O}_{1-x} \mathrm{~F}_{x}\right) \mathrm{FeAs}$, Europhys. Lett. 83, 27006 (2008).

[12] V. Cvetkovic and Z. Tesanovic, Valley density-wave and multiband superconductivity in iron-based pnictide superconductors, Phys. Rev. B 80, 024512 (2009).

[13] A. B. Vorontsov, M. G. Vavilov, and A. V. Chubukov, Superconductivity and spin-density waves in multiband metals, Phys. Rev. B 81, 174538 (2010).

[14] I. Eremin and A. V. Chubukov, Magnetic degeneracy and hidden metallicity of the spin-density-wave state in ferropnictides, Phys. Rev. B 81, 024511 (2010).

[15] A. V. Balatsky, D. N. Basov, and J.-X. Zhu, Induction of charge density waves by spin density waves in iron-based superconductors, Phys. Rev. B 82, 144522 (2010).

[16] S. Avci et al., Magnetically driven suppression of nematic order in an iron-based superconductor, Nat. Commun. 5, 3854 (2014).

[17] A. E. Böhmer, F. Hardy, L. Wang, T. Wolf, P. Schweiss, and C. Meingast, Superconductivity-induced re-entrance of the orthorhombic distortion in $\mathrm{Ba}_{1-x} \mathrm{~K}_{x} \mathrm{Fe}_{2} \mathrm{As}_{2}$, Nat. Commun. 6, 7911 (2015).

[18] L. Wang, F. Hardy, A. E. Böhmer, T. Wolf, P. Schweiss, and C. Meingast, Complex phase diagram of $\mathrm{Ba}_{1-x} \mathrm{Na}_{x} \mathrm{Fe}_{2} \mathrm{As}_{2}$ : A multitude of phases striving for the electronic entropy, Phys. Rev. B 93, 014514 (2016).

[19] J. M. Allred et al., Double-Q spin-density wave in iron arsenide superconductors, Nat. Phys. 12, 493 (2016).

[20] X. Zhu, F. Han, G. Mu, P. Cheng, B. Shen, B. Zeng, and H.-H. Wen, Transition of stoichiometric $\mathrm{Sr}_{2} \mathrm{VO}_{3} \mathrm{FeAs}$ to a superconducting state at $37.2 \mathrm{~K}$, Phys. Rev. B 79, 220512 (2009).

[21] I. I. Mazin, $\mathrm{Sr}_{2} \mathrm{VO}_{3} \mathrm{FeAs}$ as compared to other iron-based superconductors, Phys. Rev. B 81, 020507 (2010).

[22] M. Tegel, T. Schmid, T. Sturzer, M. Egawa, Y. Su, A. Senyshyn, and D. Johrendt, Possible magnetic order and suppression of superconductivity by $\mathrm{V}$ doping in $\mathrm{Sr}_{2} \mathrm{VO}_{3} \mathrm{FeAs}$, Phys. Rev. B 82, 140507 (2010).

[23] G.-H. Cao et al., Self-doping effect and successive magnetic transitions in superconducting $\mathrm{Sr}_{2} \mathrm{VFeAsO}_{3}$, Phys. Rev. B 82, 104518 (2010).

[24] S. Tatematsu, E. Satomi, Y. Kobayashi, and M. Sato, Magnetic ordering in V-layers of the superconducting system of $\mathrm{Sr}_{2} \mathrm{VFeAsO}_{3}$, J. Phys. Soc. Jpn. 79, 123712 (2010).

[25] H. Nakamura and M. Machida, Magnetic ordering in blocking layer and highly anisotropic electronic structure of high- $T_{c}$ iron-based superconductor $\mathrm{Sr}_{2} \mathrm{VFeAsO}_{3}$ : LDA + U study, Phys. Rev. B 82, 094503 (2010).
[26] J. Munevar et al., Static magnetic order of $\mathrm{Sr}_{4} \mathrm{~A}_{2} \mathrm{O}_{6} \mathrm{Fe}_{2} \mathrm{As}_{2}$ $(\mathrm{A}=\mathrm{Sc}$ and $\mathrm{V})$ revealed by Mössbauer and muon spin relaxation spectroscopies, Phys. Rev. B 84, 024527 (2011).

[27] G. Garbarino, R. Weht, A. Sow, C. Lacroix, A. Sulpice, M. Mezouar, X. Zhu, F. Han, H. H. Wen, and M. Núñez-Regueiro, Direct observation of the influence of the $\mathrm{FeAs}_{4}$ tetrahedron on superconductivity and antiferromagnetic correlations in $\mathrm{Sr}_{2} \mathrm{VO}_{3} \mathrm{FeAs}$, Europhys. Lett. 96, 57002 (2011).

[28] K. Ueshima, F. Han, X. Zhu, H.-H. Wen, S. Kawasaki, and G.-Q. Zheng, Magnetism and superconductivity in $\mathrm{Sr}_{2} \mathrm{VFeAsO}_{3}$ revealed by ${ }^{75} \mathrm{As}-$ and ${ }^{51} \mathrm{~V}-\mathrm{NMR}$ under elevated pressures, Phys. Rev. B 89, 184506 (2014).

[29] J. M. Ok et al., Frustration-driven $\mathrm{C}_{4}$ symmetric orders in a hetero-structured iron-based superconductor, arXiv:1706 .08157.

[30] F. Hummel, Y. Su, A. Senyshyn, and D. Johrendt, Weak magnetism and the Mott state of vanadium in superconducting $\mathrm{Sr}_{2} \mathrm{VO}_{3} \mathrm{FeAs}$, Phys. Rev. B 88, 144517 (2013).

[31] See Supplemental Material at http://link.aps.org/ supplemental/10.1103/PhysRevLett.119.227001 for details, which includes Ref. [32].

[32] S. Choi et al., Atomic-scale observation and manipulation of plaquette antiferromagnetic order in iron-based superconductor, arXiv:1608.00884.

[33] S. Krause, G. Herzog, A. Schlenhoff, A. Sonntag, and R. Wiesendanger, Joule Heating and Spin-Transfer Torque Investigated on the Atomic Scale Using a Spin-Polarized Scanning Tunneling Microscope, Phys. Rev. Lett. 107, 186601 (2011).

[34] Y. K. Kim et al., Possible role of bonding angle and orbital mixing in iron pnictide superconductivity: Comparative electronic structure studies of LiFeAs and $\mathrm{Sr}_{2} \mathrm{VO}_{3} \mathrm{FeAs}$, Phys. Rev. B 92, 041116(R) (2015).

[35] S. Choi et al., Correlation of Fe-Based Superconductivity and Electron-Phonon Coupling in an FeAs/Oxide Heterostructure, Phys. Rev. Lett. 119, 107003 (2017).

[36] I. I. Mazin, D. J. Singh, M. D. Johannes, and M. H. Du, Unconventional Superconductivity with a Sign Reversal in the Order Parameter of $\mathrm{LaFeAsO}_{1-x} \mathrm{~F}_{x}$, Phys. Rev. Lett. 101, 057003 (2008).

[37] R. M. Fernandes and A. V. Chubukov, Low-energy microscopic models for iron-based superconductors: A review, Rep. Prog. Phys. 80, 014503 (2017).

[38] H. Kontani and S. Onari, Orbital-Fluctuation-Mediated Superconductivity in Iron Pnictides: Analysis of the FiveOrbital Hubbard-Holstein Model, Phys. Rev. Lett. 104, 157001 (2010).

[39] T. Saito, S. Onari, Y. Yamakawa, H. Kontani, S. V. Borisenko, and V.B. Zabolotnyy, Reproduction of experimental gap structure in LiFeAs based on orbital-spin fluctuation theory: $\mathrm{s}_{++}$-wave, $\mathrm{s}_{ \pm}$-wave, and hole- $\mathrm{s}_{ \pm}$-wave states, Phys. Rev. B 90, 035104 (2014). 